\title{
Automatic Optimization of Multichip RFID tags
}

\author{
Yuanhe Xiong \\ Politecnico di Torino \\ Torino, Italy \\ yuanhe.xiong@polito.it \\ Gaetano Marrocco \\ DISP-University of \\ Roma Tor Vergata, \\ Rome, Italy \\ marrocco@disp.uniroma \\ 2.it
}

\author{
Stefano Caizzone \\ Institute of \\ Communications and \\ Navigation, \\ German Aerospace \\ Center(DLR), \\ Wessling, Germany \\ Giuseppe Vecchi \\ Politecnico di Torino \\ Torino, Italy \\ giuseppe.vecchi@polito.it
}

\author{
Cecilia Occhiuzzi \\ DISP-University of \\ Roma Tor Vergata, \\ Roma, Italy \\ occhiuzzi@disp.uniroma2.it \\ Mario Orefice \\ Politecnico di Torino \\ Torino, Italy \\ mario.orefice@polito.it
}

\begin{abstract}
The automatic optimization is proposed of the passive RF part of RFID, with special attention to multi-chip tags, and to the novel concept of RFID grids. Performance metrics follows a recent all-comprehensive approach. The proposed approach employs a Genetic Algorithm-based optimization, and an efficient electromagnetic problem parameterization and solution strategy. Resulting structures, while non-intuitive in shape, exhibit enhanced performance.
\end{abstract}

\section{INTRODUCTION}

The radiofrequency identification technology (RFID) for object tagging [1] is rapidly evolving toward the "Internet of Things" [2,3]: the convergence of a number of research disciplines (identification, real-time localization, sensor networks, pervasive computing) that enable the Internet to get into the real world of physical objects interacting with web services.

The challenges to the design of present- and next-generation RFID have spurred a significant research over the past few years, and the availability of low-cost chips has prompted the study of multi-chip tags.

In this context, electromagnetic (EM) theory has recently revamped antenna and radiation concepts into expanding the capabilities of RFID. An interesting such example is the RFID grid paradigm recently appeared [3].

\section{TAG FUNCTIONAL OPTIMIZATION}

Recent embodiments of multi-chip, and grid types of tags have shown the necessity of optimizing the EM properties of the passive part of the tag, also for performance robustness.

Along this line, we have undertaken an effort to design this passive part via automatic optimization, yet keeping the information on the chip(s) directly in the process (linear behavior considered, as usual). Antenna optimization has been a constant effort in the last years and various efficient methods have been developed due to the whole community's brilliant work; we employ here the approach devised in [4].

This approach is especially useful for electrically small antennas, and since it considered multi-port structures and reconfigurability is well suited for the present endeavor.

The passive part (metallization) of the multi-chip tag is automatically designed to optimize the global parameters introduced and discussed in [3], rather than individual radiation or (multi-port) impedance matching, which is a novel approach.

Consistency checks against previous partial optimization of tags [5] haven been preliminarily carried out.

The obtained structures show detailed structure, as often with automatic optimization, and efforts were done to keep complexity within fabrication process requirement.

\section{GENETIC ALGORITHM AND METHOD OF MOMENTS}

During the past several decades, genetic algorithm (GA) has been involved more and more in electromagnetic optimization applications, especially in multi-objective cases. It's a stochastic global search method based on Darwin's theory of evolution and has several advantages over the traditional optimization approaches, such as conjugate gradient and random search [6]. J. Michael Johnson et al. [7] gives a vivid description on the principles of GA, as well as several application examples in electromagnetics.

Electric field integral equation (EFIE) and Method of moments (MoM) solution is a classical way of dealing with EM radiation and scattering problems as well as RF circuits, which focus primarily on the solution of surface current. Once the structure is discretized in a computational mesh, and basis functions defined over it, the integral equation yields a linear system

$$
Z * I=V
$$

where $Z$ is the system matrix and I are the unknown coefficients of the basis function expansion. The common choice is using a triangular grid and the Rao-Wilton-Glisson (RWG) basis functions [8], which is the approach we employ during our work.

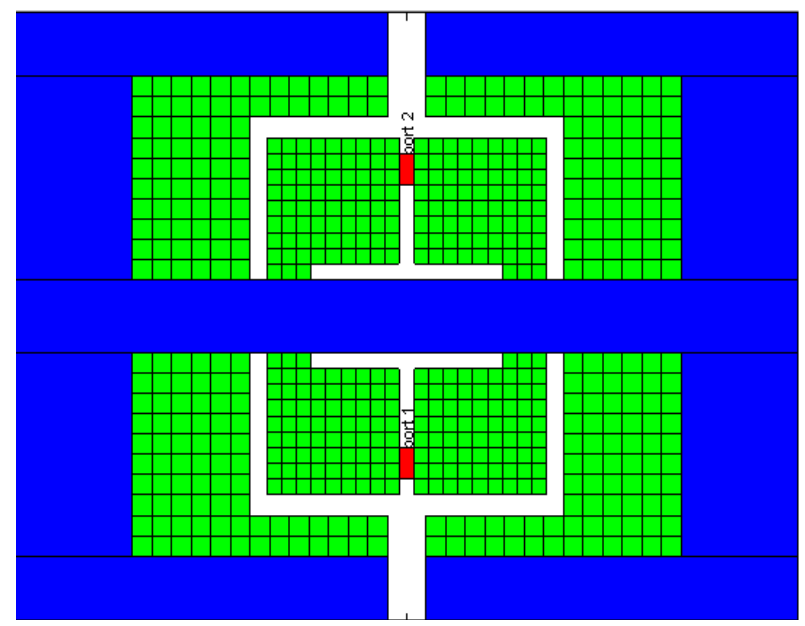

FIGURE 1- TOPOLOGY OF THE MOTHER RFID ANTENNA STRUCTURE

In this contribution, an integration of MoM/EFIE and GA is employed to design and optimize the RFID tag antenna under some specific constraints. This approach originates from [4,9], where $\mathrm{MoM} / \mathrm{EFIE}$ is applied to analyze the EM characteristics of antenna 
structures, while GA provides the capability of searching for optimal structures.

Besides, two techniques which can improve highly the efficiency of the GA optimization process are posted, the direct-matrix manipulation (DMM) and matrix partitioning. By means of the first technique, the system matrix fill-in is done only once and for all during the whole optimization process, this is realized thanks to the introduction of the concept "mother" structure. All the sub-structures (individuals) generated during the optimization process can be obtained by removing a certain part of the mother structure, which means that for any individual, its system matrix can be configured via taking away the corresponding rows and columns from the system matrix of the mother structure; much more efficient compared with the traditional perception that each structure needs a system matrix fill-in. An example of this is shown in Figure 1; we note that the geometric shape optimization here employs rectangular "pixels" for geometric convenience, while EM analysis is carried out on underlying triangles. The second trick takes into consideration the fact that within an antenna optimization problem, a certain portion of the antenna may be unmodified during the whole optimization process. Therefore, instead of processing (operations like LU decomposition or direct matrix inversion) the whole system matrix, we partition it into 4 blocks and use partial inversions.

The basic idea behind our work is based on the above theoretical analysis. Once the surface current is solved, further calculations can be carried out to obtain quantities we are interested in, such as radiation pattern, input impedance, radar cross section, as well as return loss and so on.

\section{MULTI-PORT RFID TAG ANTENNA}

In [3], the concept "grid" is introduced, which represents a generally coupled multitude of UHF tags, including standalone tags in close mutual proximity as well as tags with a multiplicity of embedded RFID microchip transponders able to achieve advanced capabilities such as redundancy and sensing; also it's fully investigated as an EM interconnected system. In our paper, we take the latter formation of grid, i.e., a tag with multiple chips connected to it; this tag can be handled as a multi-port antenna. Without loss of generality, we take a two-port antenna for analysis.

With respect to RFID tag antenna design, dipole-like antenna is always preferred as a choice due to its simplicity, light weight, as well as low cost. The prototype of the tag antenna is shown in Figure 1 , which derives from [5]; this antenna can be applied to monitor the temperature in an environment like refrigeration house where the temperature should be kept below a pre-defined threshold. The working principle of this two-port antenna is simple. One of the ports of the antenna is always active (in our work, port 1), thus it can receive the signals from reader and backscatter the corresponding information to the reader all the time; the other port, attached by a shape-memory alloy, undergoes the following scenario: when the temperature is lower than the threshold, the shape-memory alloy connects the port, therefore a parallel connection of both tag and alloy impedance should be considered here; while when the temperature goes beyond the threshold, the alloy gets disconnected, thus this port works the same way as the always active port. The reader can make a decision based on whether the information it collects changes or not.

A typical design goal of RFID antenna is the maximum power transmission between the chip and the tag antenna, which requires a complex conjugate between the port's input impedance and the chip impedance. Another quantity we emulate here is the newly developed concept with the name embedded realized gain [3]; it differs from the standard tag configuration, since it successfully confuses together impedance matching, gain and polarization. This parameter is able to completely characterize the power scavenging performances of a RFID grid in free space [10], which is equivalent to maximize the reading distance of the grid.

\section{SIMULATION AND RESULTS}

GA along with the MoM/EFIE technique is employed in our work to automatically minimize the objective function, which offers a much greater search space and flexibility, apart from the fact that it can provide potentially better and more robust resulting structures.

\subsection{Optimization Procedure}

Our work is divided mainly into two parts. Both of the two parts share the same optimization procedure, which is explained below and illustrated in Figure 2.

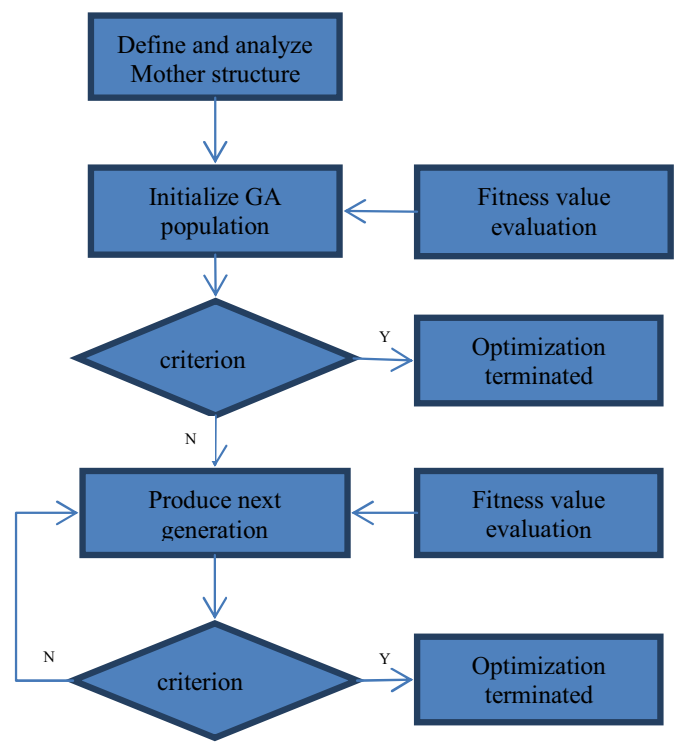

FIGURE 2- FLOWCHART OF OPTIMIZATION PROCEDURE

Firstly, a mother structure is defined in Figure 1. The antenna is placed in the $x=0$ plane, with the center located in the origin, the dimension of the antenna plane is $80 \times 58 \mathrm{~mm}^{2}$, metal covers the blue and green areas, and chips will be placed in the red regions. Its system matrix is computed by employing RWG basis functions [8]. Secondly, the structure is coded with a binary coding plan. The blue part of the mother structure is treated as the constant group and will be neglected when we code the structure. Thus we need only to code the green area, which is divided into square cells. Appropriate assignment of constant area can accelerate the search for optimal individuals, since the sample space decreases if a certain part becomes unmodified during the whole optimization process. Generally, areas relatively far from the port locations are preferred choices as constant region, since the contribution to far field is dominated by surface current in the areas in proximity to the ports. Thirdly, define the GA parameters, which include generation number, individuals per generation, selection strategy, crossover rate and mutation rate and so on. Besides, the first generation is generated and its fitness value is evaluated. Fourthly run the GA optimizer. Check whether the fitness value of first generation meets the termination criterion, if yes, the optimizer stops, or else GA operations (parent selection, crossover, mutation and elitism) are performed to yield the next generation. When the next generation is produced successfully, repeat the following steps until the stop criterion is satisfied, i.e., fitness value evaluation, stop criterion check, offspring generation (if stop criterion is still not met) or optimizer termination (stop criterion is met). 


\subsection{Impedance Matching Optimization}

When developing the objective function for optimizing impedance matching problem, one should take into consideration both working scenarios. For the below-threshold case, we are glad to see the situation that the input impedance of the active port is complex conjugate with the chip impedance (in this case we can achieve maximum power transmission), while the input impedance of the inactive port in parallel with the alloy's impedance is extremely mismatched with the chip impedance (ideally, no power transferred to the chip via the tag antenna); for the above-threshold case, since the alloy gets disconnected with the port, the situation that both input impedance of the two ports are complex conjugate of the chip impedance is desired. One can refer to [5] for more detailed information about the formation of the objective function, which we inherit in our work.

The existence of gaps (white) in mother structure makes this antenna resembles the final design in [5], which takes advantage of dipole-like structures and $\mathrm{T}$-match, the difference between them is that, the mother structure in our work is covered by metal (modeled as PEC) almost in the whole antenna plane, which undoubtedly enlarges the probability of finding better-suited structures. The chip we adopt in our work is NXP TSS OP8 with nominal input impedance at $869 \mathrm{MHz} Z_{\text {chip }}=15-j 135 \Omega$.

There are in total 596 squares in the mother structure. In the GA we apply, 60 iterations (generations) are run, with 250 individuals in each generation, the mutation rate is 0.003 , a scatter crossover plan, which is similar with proportionate selection, is used, elitism is also included in the implementation. The GA will automatically search for an optimal structure as it proceeds.

The convergence curve of fitness value versus number of generations is presented in Figure 3. A similar EM characteristics analysis is applied to the final design in [5], whose fitness value is found to be around 20 after computation. From Figure 3, we can see that the GA can evolve towards much smaller fitness value, hence better structures; the monotonically decreasing feature of the curve is due to the introduction of elitism, which ensures that the smallest fitness value in the next generation will be no larger than the corresponding value in the previous generation.

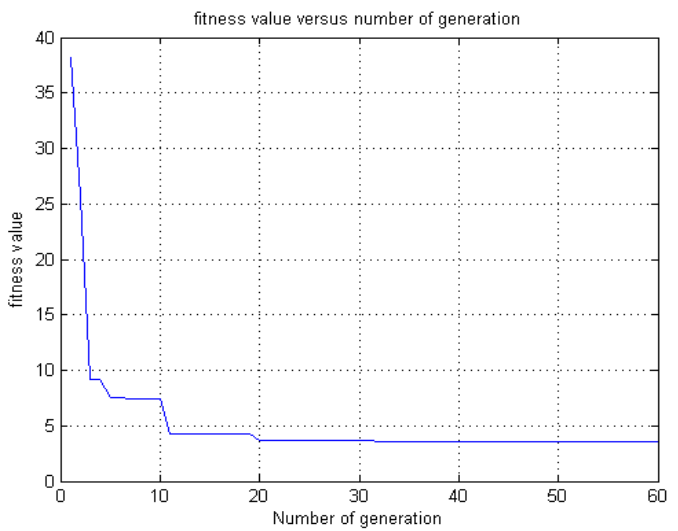

FIGURE 3- CONVERGENCE OF FITNESS VALUE VERSUS GENERATION NUMBER

Based on the above results, we can come to the conclusion that GA along with MoM/EFIE can lead to well-suited structures which meet the objective function defined in [5] very well.

\subsection{Embedded Realized Gain Optimization}

In this section, we will study the effect of GA when used to optimize the quantity embedded realized gain. The frequency band we are interested in lies in the UHF band, from $840 \mathrm{MHz}$ to $960 \mathrm{MHz}$. The nature of this parameter enables it being taken into consideration within a multi-chip antenna optimization. The definition of this parameter is given in the following expression,

$$
\widetilde{G_{n}}=4 \eta_{0} R_{C, n}\left|\left[\mathbf{Y}_{G}\right]_{n} \cdot \mathbf{g}\right|^{2}
$$

where subscript $n$ stands for the $\mathrm{n}$-th port of the structure, $\eta_{0}$ is the characteristic impedance in free space, the detailed meanings of the other symbols can be found in $[3,10]$.

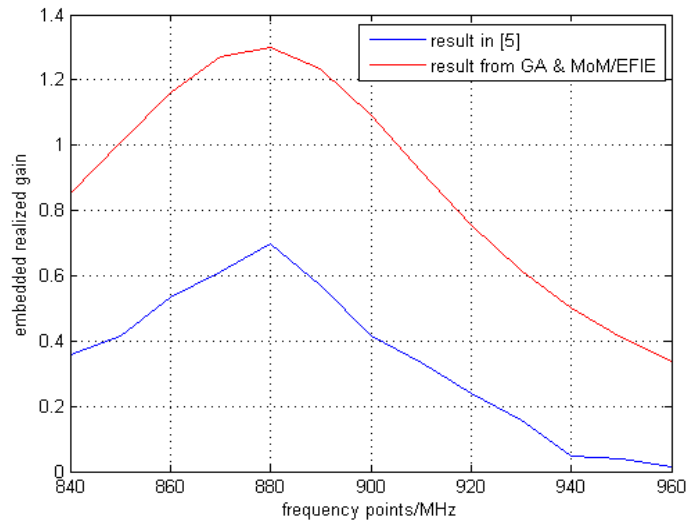

FIGURE 4- EMBEDDED REALIZED GAIN COMPARISON

The major difference between this section and the previous one lies in the objective function. Here, we emphasize the maximization of embedded realized gain in some desired directions over the whole frequency band, with all ports taken into account. For sake of simplicity, only one port (port 2) and one direction (theta and phi components are both 90 degree) are considered. The objective function for this problem is given in equation (3).

$$
\text { fitness }=\max \left(\widetilde{G_{n f}}\right), \forall n f
$$

where $n f$ means frequency points. We evenly select 13 frequency points in the band, with a step of $10 \mathrm{MHz}$.

Simulations have been run and one of the optimal results is presented in Figure 4. Substantial improvements can be observed, compared with what's presented in [10], which is what we desire through using the above optimization procedure. The approach can be extended further to solve more complicated problems, where the embedded realized gain for several observation angles along with several ports over the frequency band should all be considered.

\subsection{Structure Complexity Reduction}

Based on the way we discretize the mother structure, GA may, and very often, yield non-intuitive structures, as what is shown in the above picture in Figure 5. Although these non-intuitive results can satisfy the objective function pretty well, they are not so convenient when it comes to manufacturing. Some efforts have been done to reduce the complexity of the GA's output. Basically, the complexity can be lowered from two aspects, both under the assumption that the EM performance of the structure should not decrease much before and after complexity reduction. The first aspect takes an eye on the holes present in the output structure, especially holes occupying only one square, take Figure 5 as an example, compare the two graphs, we can find four black squares present in the structure for fabrication, while correspondingly they are white squares present in the above structure, which means that they are not part of the individual, the addition of these black squares makes the structure more smooth. For the second aspect, we would like to delete those isolated squares present in GA's output individual, especially those with only one square; we can find four cyan squares present in the fabrication model in Figure 5, and they can be seen also in the GA's output individual, however, since they are isolated, it would be better to delete them to reduce the structural complexity. The two aspects are independent of each other. Comparisons have been done between the two structures in Figure 5, and it turns out that they perform closely to each other, which is the effect we desire. 


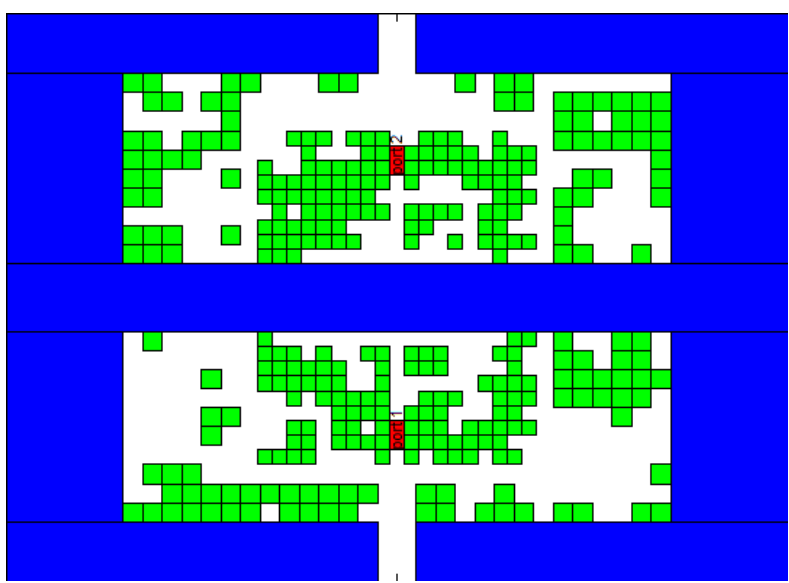

STRUCTURE AFTER OPTIMIZATION

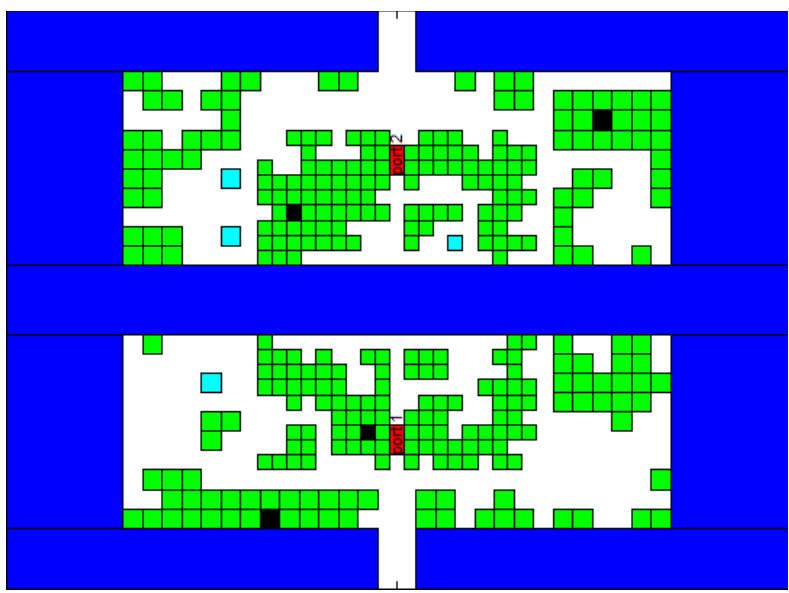

STRUCTURE FOR FABRICATION

FIGURE 5- OPTIMAL STRUCTURE AND THE ONE FOR FABRICATION

\section{CONCLUSIONS}

Automatic optimization of RFID tag antennas is presented in our work. The approach features a combination of GA and MoM/EFIE, in which MoM/EFIE is employed to analyze the EM properties of antenna and GA is applied to search for optimal solution. The theories behind this method are explained explicitly and detailed procedures of implementation are illustrated. Two examples sharing the same initial RFID antenna structure are provided, one for impedance matching optimization, while the other one for the perfection of embedded realized gain, a newly developed concept for RFID grid. Satisfying outputs have been achieved for both examples, which verify the feasibility of our optimization approach. Besides, efforts for smoothing the non-intuitive structure have been introduced, which help reduce the structure complexity and thus make the antenna more suited for manufacture purpose.

\section{ACKNOWLEDGEMENTS}

This work was sponsored by Italian MIUR program PRIN 2008 through the grant 2008CRSHTE_004 (MULTI-TAG: Electromagnetic technology for multiple-interrogation active and passive RFID systems).

\section{REFERENCES}

[1] D. M. Dobkin, The RF in RFID: Passive UHF RFID in Practice. Amsterdam: Elsevier, 2007.
[2] D. Preuveneers and Y. Berbers, "Internet of Things: A contextawareness perspective," in The Internet of Things: From RFID to the Next-Generation Pervasive Networked Systems, L. Yan, Y. Zhang, L. T. Yang, and H. Ning, Eds. London: Auerbach Publications, 2008.

[3] G. Marrocco "RFID Grids: Part I-Electromagnetic Theory", IEEE TRANSCATIONS ON ANTENNAS AND PROPAGATION, VOL. 59, NO. 3, MARCH 20111019.

[4] J. L. Araque Quijano and G. Vecchi, "Optimization of an innovative type of compact frequency-reconfigurable antenna," IEEE Transactions on Antenna and Propagation, vol. 57, pp. 918, Jan. 2009.

[5] S. Caizzone, C. Occhiuzzi and G. Marrocco, "Multi-Chip RFID Antenna Integrating Shape-Memory Alloys for Detection of Thermal Thresholds", TRANSCATIONS ON ANTENNAS AND PROPOGATION, VOL. 59, 2011.

[6] J. Michael Johnson and Yahya Rhamat-Samii, "Genetic Algorithm in Engineering Electromagnetics", IEEE Antennas and Propagation Magazine, vol. 39, issue 4, pp. 7-21.

[7] R. L. Haupt, D. H. Werner, "Genetic Algorithm in Electromagnetics”, Wiley, 2007. ISBN 978-0-471-48889-7.

[8] S. M. Rao, D. R. Wilton, and A. W. Glisson, "Electromagnetic scattering by surfaces of arbitrary shape", IEEE Trans. Antennas Propagat., vol. AP-30, pp409-418, May 1982.

[9] J. Michael Johnson and Yahya Rhamat-Samii, "Genetic Algorithm and Method of Moments (GA/MoM) for the Design of Integrated Antennas", IEEE TRANSACTIONS ON ANTENNAS AND PROPAGATION, VOL. 47, NO. 10, OCTOBER 1999.

[10] S. Caizzone, G. Marrocco "RFID Grids: Part IIExperimentations", IEEE TRANSCATIONS ON ANTENNAS AND PROPAGATION, VOL. 59, NO. 8, AUGUST 20111019. 